\title{
ARTICLE
}

\section{Accuracy of IOL power calculations in the very elderly}

\author{
Ruti Sella ${ }^{1} \cdot$ Linda Chou $^{1} \cdot$ Alexander K. Schuster $^{2} \cdot$ Helena E. Gali $^{1} \cdot$ Robert N. Weinreb $^{1} \cdot$ Natalie A. Afshari $^{1}$
}

Received: 23 October 2019 / Revised: 12 December 2019 / Accepted: 13 December 2019 / Published online: 13 January 2020

(c) The Author(s), under exclusive licence to The Royal College of Ophthalmologists 2020

\begin{abstract}
Background/objectives To analyze the refractive predictability and outcomes of cataract surgery in the very elderly ( $\geq 85$ years old).

Subjects/methods A retrospective case-series performed at the Shiley Eye Institute, University of California San Diego, USA. Electronically pulled data of 2444 surgeries revealed 147 surgeries on 133 very elderly patients. Chart review was conducted for all very elderly and corresponding control patients (75-84 years old). The first operated eyes of patients with final best-corrected visual acuity $\geq 20 / 40$, axial length (AL) 22-26 mm, and implanted SN60WF IOL were included. Patients with ocular comorbidities and/or intra- or post-operative complications were excluded. Prediction errors of refractive outcome and percentage of eyes within $\pm 0.50 \mathrm{D}$ and $\pm 1.00 \mathrm{D}$ were compared between the groups for the Holladay 1 and Barrett Universal II (Barrett) formulas. Logistic regression analysis for achievement of $\pm 1.00 \mathrm{D}$ was conducted.

Results Final analysis included 90 eyes ( $n=44$, very elderly, $n=46$, control patients). Median absolute refractive error (MedAE) with Holladay 1, but not Barrett formula, was significantly higher in the older group $(p=0.02$ and $\mathrm{p}=0.07$, respectively). The MedAE in the older group was lower using the Barrett compared to Holladay $1(p=0.02)$. Fewer older patients than younger patients achieved refraction within $\pm 0.50 \mathrm{D}$ and $\pm 1.00 \mathrm{D}$ from goal, using the Holladay $1(p=0.049$ and $p=0.002$ respectively). Logistic regression analysis supported the relationship between Holladay 1 predictive refractive error of $>1.00 \mathrm{D}$ and patient's age $(p=0.046)$.

Conclusions Very elderly patients undergoing cataract surgery may be prone to reduced refractive precision, particularly with utilization of the Holladay 1 formula.
\end{abstract}

\section{Introduction}

With increased aging of the world's population, the very elderly (aged $\geq 85$ ) [1] are now expected to constitute a higher percentage of patients undergoing cataract surgery, and this number is expected to rise [2]. Few previous studies evaluating the outcomes of cataract surgery have focused on this subpopulation. Of these studies, most have compared the safety profile of cataract surgery in these very elderly patients to their younger counterparts [3-5] or have focused on visual outcomes and quality of

Natalie A. Afshari

naafshari@ucsd.edu

1 Shiley Eye Institute, Viterbi Family Department of Ophthalmology, University of California San Diego, La Jolla, CA, USA

2 Department of Ophthalmology, University Medical Center of the Johannes Gutenberg University Mainz, Mainz, Germany life after surgery [5-9]. While acknowledging that comorbidities like wet age-related macular degeneration or advanced glaucoma may limit visual outcomes in this subpopulation, these studies have shown that cataract surgery is safe in this age group $[3,10,11]$ and leads to improvement of patients' daily functioning and quality of life measures [8, 11, 12]. More patients than ever before reach their $80 \mathrm{~s}$ with good general and ocular health, as they are still mobile and continue daily routines and recreational activities. This increase in years of good health warrants fully addressing refractive expectations as well.

Older age has been associated with collagen structural changes impacting scleral fiber alignment and matrix stiffness [13] as well as stretching qualities of the anterior capsule and lens zonule. This changes elastic properties over time [14, 15]. Moreover, age has been shown to affect the morphology of the Schlemm's canal (SC) and trabecular meshwork (TM) [16]. Evidence of changes of the anterior chamber depth (ACD) have also been reported in the elderly [17]. 
While studies indicate that refractive results in the very elderly are overall good $[11,18]$ and that a patient's age may impact intraocular lens (IOL) prediction error of the SRK/T formula $[19,20]$, there has been no attempt to assess refractive predictabilities of more modern IOL calculation formulas in this specific age group. We therefore analyzed the accuracy of IOL calculations in the very elderly in comparison to their younger counterparts (aged 75-84) using two widely used formulas: the Holladay 1 and the Barrett Universal II.

\section{Materials (subjects) and methods}

This study was performed in a single tertiary setting at the Shiley Eye Institute, University of California, San Diego. Prospective Institutional Review Board (IRB) approval was granted for this study by the IRB office of University of California San Diego, and it conformed to the tenets of the Declaration of Helsinki.

\section{Patient selection}

Figure 1 summarizes patient selection criteria for this study. A total of 7090 cataract surgeries were performed by experienced surgeons of cornea and glaucoma services of the Shiley Eye Institute, University of California, San Diego, between January 2015 and May 2018. Power calculation for this study was based on the two-sided Mann-Whitney- $U$ test in median absolute refractive error (MedAE) between very elderly ( $\geq 85$ years old) patients and the younger (75-84 years old) patients (controls). With a calculated minimal sample size of $n=88$ (44 in each group), this test was expected to have $80 \%$ power at the 0.05 significance level to detect an effect size of $d=0.6$ between the groups. Internal data query showed 425/ 7090 surgeries $(6 \%)$ were performed on patients $\geq 85$ years old. Data was electronically queried solely for surgeries performed on the first two days of each week, yielding 2444 surgical cases, out of which 147 surgeries were performed on the very elderly.

After exclusion of all second eye surgeries, 133 patient charts in the very elderly group and their paired surgical date consecutive 75-84-year-old patients were reviewed. Patient demographic data were collected as well as each eye's preoperative manifest refraction and biometry measurements including $\mathrm{ACD}$, keratometry $(\mathrm{K})$ values, axial length (AL), and selected IOL power. The ACD was defined as axial distance from the corneal epithelium to lens. IOL calculations were performed with the Holladay 1 and the Barret Universal II formulas, and the predictive refractive error (RE) of each formula using the IOL power that was implanted was documented. Postoperatively, best spectacle-corrected visual acuity (BCVA) and stable manifest refraction were documented. Refractive and absolute refractive errors for each formula, in addition to the percentage of eyes within $\pm 0.50 \mathrm{D}$ and $\pm 1.00 \mathrm{D}$ of the predicted refraction of the IOL that was implanted were compared between the age groups.

In adherence with the Hoffer et al. protocols for studies of intraocular lens formula accuracy [21, 22], only patients with a final BCVA better than 20/40, an AL of 22-26 mm, who all underwent implantation of the same model of IOL (SN60WF, Alcon Laboratories, Inc., Fort Worth, TX) were included. All included patients underwent ocular biometry 1 month prior to surgery, using the partial coherence interferometer (IOLMaster, V. 5.2.1, Carl Zeiss Meditec, Inc., Dublin, CA), and a corneal tomography exam (Pentacam HR, Version 6.08r30; Oculus, Wetzlar, Germany). When AL measurements were not achievable or when a difference of $\geq 0.3 \mathrm{~mm}$ in the measured AL between the eyes was noted, immersion ultrasound biometry was performed. All included patients had a documented follow-up visit 1-3 months post operatively, at which time a manifest refraction was performed by a licensed optometrist. Exclusion criteria included patients with partial follow-up data (missing stable refraction information, lost to follow-up, or missing charts), low quality of biometry measurements defined by a signal-to-noise ratio $(\mathrm{SNR}) \leq 2$ by the IOLMaster, eyes with significant ocular comorbidities, history of refractive surgery, and/or intra- or postoperative complications.

\section{Intraocular lens calculations}

All cataract surgeries involved a temporal clear corneal incision, phacoemulsification, and implantation of an IOL into the capsular bag. IOL choice was made at the discretion of the surgeon. IOL calculations output based on biometry using the Holladay 1 formula and the Barrett Universal II (v1.05, available at http://calc.apacrs.org/barrett_universa 12105) were documented in all cases. The nominal Aconstant (118.7) for the SN60WF IOL and Surgeon Factor for the Holladay 1 formula (1.84) were optimized for the Zeiss IOLMaster as specified by the User Group for Laser Interference Biometry (ULIB) website (available online at: http://ocusoft.de/ulib) and were not optimized per surgeon. Predicted refractive error (PE) was defined as the difference between the RE, the formula predicted, and the patient's actual stable refraction postoperatively [21]. No adjustment of the PE to zero was applied in this study due to a very low mean refractive $\mathrm{PE}$ in both groups $(0.15 \mathrm{D}$ in each of the groups) [23]. Moreover, this study reflects the current practice in many centers today where personalization of the lens constant is not routinely applied.

To further validate our calculations, we used the online lens formula performance audit calculator (Version 1.6, 


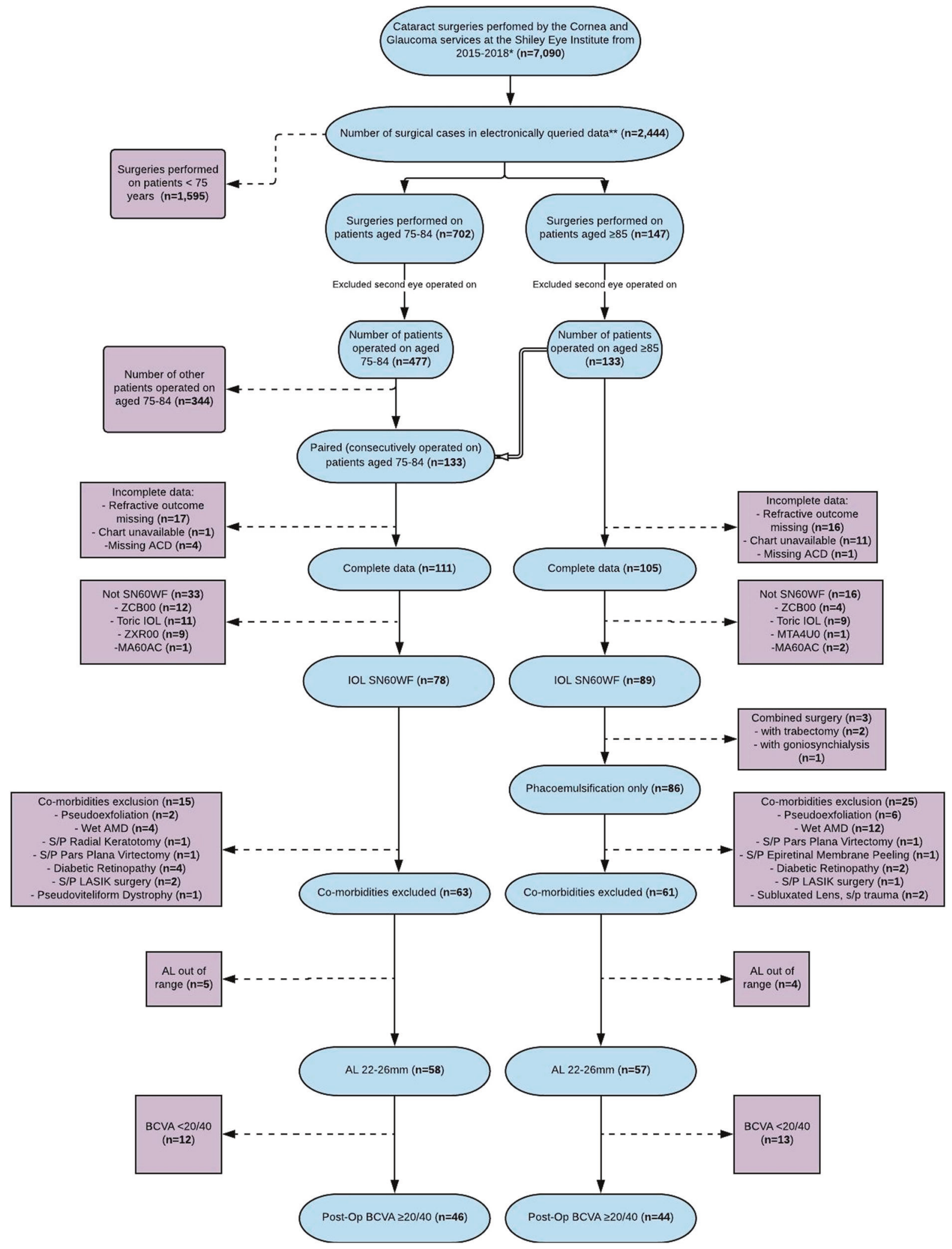

Fig. 1 Summary of criteria for patient selection. Asterisk indicates January 2015 until July 2018. Double asterisks indicate that total number of cases queried electronically was based on power analysis and internal data indicating that $6 \%$ of total cataract surgeries at the
Shiley Eye Institute were performed on patients over the age of 85 . ACD anterior chamber depth, IOL intraocular lens, AL axial length, BCVA best-corrected visual acuity, S/P status post, AMD age-related macular degeneration. 
available online at http://saurabhsawhney.wix.com/calcula tors; http://www.insighteyeclinic.in/SIA_calculator.php).

Final analysis included 44 patients in the very elderly group and 46 patients in the younger group.

\section{Statistical analysis}

Baseline AL, ACD, and mean $\mathrm{K}$, as well as biometric SNR, baseline BCVA, post-operative BCVA, and the improvement of BCVA and the MedAE with each of the formulas were compared between the age groups using the two-sided nonparametric Mann-Whitney test. The percentage of eyes within a refractive goal of $\pm 0.50 \mathrm{D}$ and $\pm 1.00 \mathrm{D}$ of the predicted refraction from the IOL that was implanted was compared between the age groups using the Fisher's exact test for each formula. The two-sided Wilcoxon matched-pairs signed rank test was used to compare the MedAE in the very elderly group between the Holladay 1 and the Barrett formulas. Multiple logistic regression analysis for the achievement of $\pm 1.00 \mathrm{D}$ was carried out including age, sex, mean corneal power, ACD, and AL. A $p$ value $<0.05$ was considered statistically significant.

\section{Results}

Table 1 summarizes patients' preoperative collected data. The two age groups did not differ in mean keratometry values, BCVA prior to surgery, biometry axial lens SNR, or AL. ACD was lower in the very elderly group compared to the controls $(p=0.02)$.

Postoperatively, logMAR BCVA was $0.13 \pm 0.10$ in the very elderly group, and $0.11 \pm 0.09$ in the younger group $(p=0.435)$. LogMAR BCVA improved by $0.35 \pm 0.39$ in the very elderly group and by $0.28 \pm 0.29$ in the younger group $(p=0.291)$. Data summarizing refractive outcomes compared to predicted RE with each of the two formulas are presented in Table 2.

MedAE with the Holladay 1 formula but not with the Barrett Universal II formula was significantly higher in the very elderly group in comparison to the younger group ( $p=0.02$ and $p=0.07$, respectively). The MedAE within the very elderly group was lower using the Barrett Universal II formula in comparison to the Holladay 1 formula $(p=0.02)$.

Table 1 Demographics and preoperative characteristics of the two age groups.

\begin{tabular}{|c|c|c|c|c|c|c|}
\hline \multirow[t]{3}{*}{ Age (years) } & \multicolumn{3}{|l|}{$75-84$} & \multicolumn{3}{|l|}{$\geq 85$} \\
\hline & $n(\%)$ & Mean \pm SD & Min-Max & $n(\%)$ & Mean \pm SD & Min-Max \\
\hline & - & $78.24 \pm 2.93$ & $75-84$ & & $86.75 \pm 2.08$ & $85-95$ \\
\hline \multicolumn{7}{|l|}{ Sex } \\
\hline Female & $33(72 \%)$ & - & - & $24(55 \%)$ & - & - \\
\hline Male & $13(28 \%)$ & - & - & $20(45 \%)$ & - & - \\
\hline OD & $24(52 \%)$ & - & - & $18(41 \%)$ & - & - \\
\hline OS & $22(48 \%)$ & - & - & $26(59 \%)$ & - & - \\
\hline $\mathrm{AL}(\mathrm{mm})$ & - & $23.67 \pm 0.89$ & $22.06-25.89$ & - & $23.86 \pm 0.95$ & $22.11-25.72$ \\
\hline Average K (D) & - & $44.05 \pm 1.74$ & $40.68-48.81$ & - & $43.61 \pm 1.49$ & $40.96-46.08$ \\
\hline $\mathrm{ACD}(\mathrm{mm})$ & - & $3.09 \pm 0.34$ & $2.49-3.77$ & - & $2.89 \pm 0.36^{\ddagger}$ & $2.20-3.57$ \\
\hline IOL power (D) & - & $20.93 \pm 2.04$ & $16.50-24.50$ & - & $21.08 \pm 2.57$ & $15.00-25.50$ \\
\hline BCVA prior surgery (logMAR) & - & $0.39 \pm 0.32$ & $0.00-2.00$ & - & $0.48 \pm 0.43$ & $0.10-2.00$ \\
\hline BCVA post surgery (logMAR) & - & $0.11 \pm 0.10$ & $0.00-0.30$ & - & $0.13 \pm 0.10$ & $0.00-0.30$ \\
\hline Biometry SNR & - & $215.40 \pm 124.61$ & $28.50-560.70$ & & $207.02 \pm 95.34$ & $90.40-386.20$ \\
\hline
\end{tabular}

$A L$ axial length, mean $K$ mean keratometry, $A C D$ anterior chamber depth, $I O L$ intraocular lens, $B C V A$ best-corrected visual acuity, $S N R$ signal noise ratio

${ }^{\ddagger} \mathrm{ACD}$ was significantly lower in the older group in comparison to the younger group $(p=0.0186)$

Table 2 Predictive refractive error per two intraocular lens calculation formulas compared by age at time of surgery.

\begin{tabular}{|c|c|c|c|c|c|c|c|c|c|c|c|c|c|c|c|c|}
\hline \multirow[b]{3}{*}{ Age } & \multirow{2}{*}{\multicolumn{2}{|c|}{ Mean error $(D) \pm S D$}} & \multirow{2}{*}{\multicolumn{2}{|c|}{$\begin{array}{l}\text { Median absolute } \\
\text { error (D) }\end{array}$}} & \multicolumn{6}{|c|}{$\%$ of eyes } & \multirow{2}{*}{\multicolumn{2}{|c|}{$\begin{array}{l}\text { Maximal plus } \\
\text { error (D) }\end{array}$}} & \multirow{2}{*}{\multicolumn{2}{|c|}{$\begin{array}{l}\text { Maximal minus } \\
\text { error (D) }\end{array}$}} & \multirow{2}{*}{\multicolumn{2}{|c|}{$\begin{array}{l}\text { Range of } \\
\text { error (D) }\end{array}$}} \\
\hline & & & & & \multicolumn{2}{|c|}{ Within $\pm 0.50 \mathrm{D}$} & \multicolumn{2}{|c|}{ Within $\pm 1.00 \mathrm{D}$} & \multicolumn{2}{|c|}{ Beyond $\pm 2.00 \mathrm{D}$} & & & & & & \\
\hline & $75-84$ & $\geq 85$ & $75-84$ & $\geq 85$ & $75-84$ & $\geq 85$ & $75-84$ & $\geq 85$ & $75-84$ & $\geq 85$ & $75-84$ & $\geq 85$ & $75-84$ & $\geq 85$ & $75-84$ & $\geq 85$ \\
\hline Holladay 1 & $-0.10 \pm 0.42$ & $-0.12 \pm 0.69$ & 0.28 & $0.48^{\ddagger}$ & 74.00 & $52.00^{\ddagger}$ & 100.00 & $82.00^{\ddagger}$ & 0.00 & 0.00 & 0.79 & 1.32 & -1.00 & -1.91 & 1.79 & 3.23 \\
\hline $\begin{array}{l}\text { Barrett } \\
\text { Universal II }\end{array}$ & $0.00 \pm 0.46$ & $0.05 \pm 0.70$ & 0.31 & 0.39 & 78.00 & 66.00 & 98.00 & 86.00 & 0.00 & 0.00 & 0.97 & 1.89 & -1.38 & -1.56 & 2.34 & 3.45 \\
\hline
\end{tabular}

$D$ diopters, $S D$ standard deviation

${ }^{\ddagger} p<0.05$ for value compared between the two age groups 

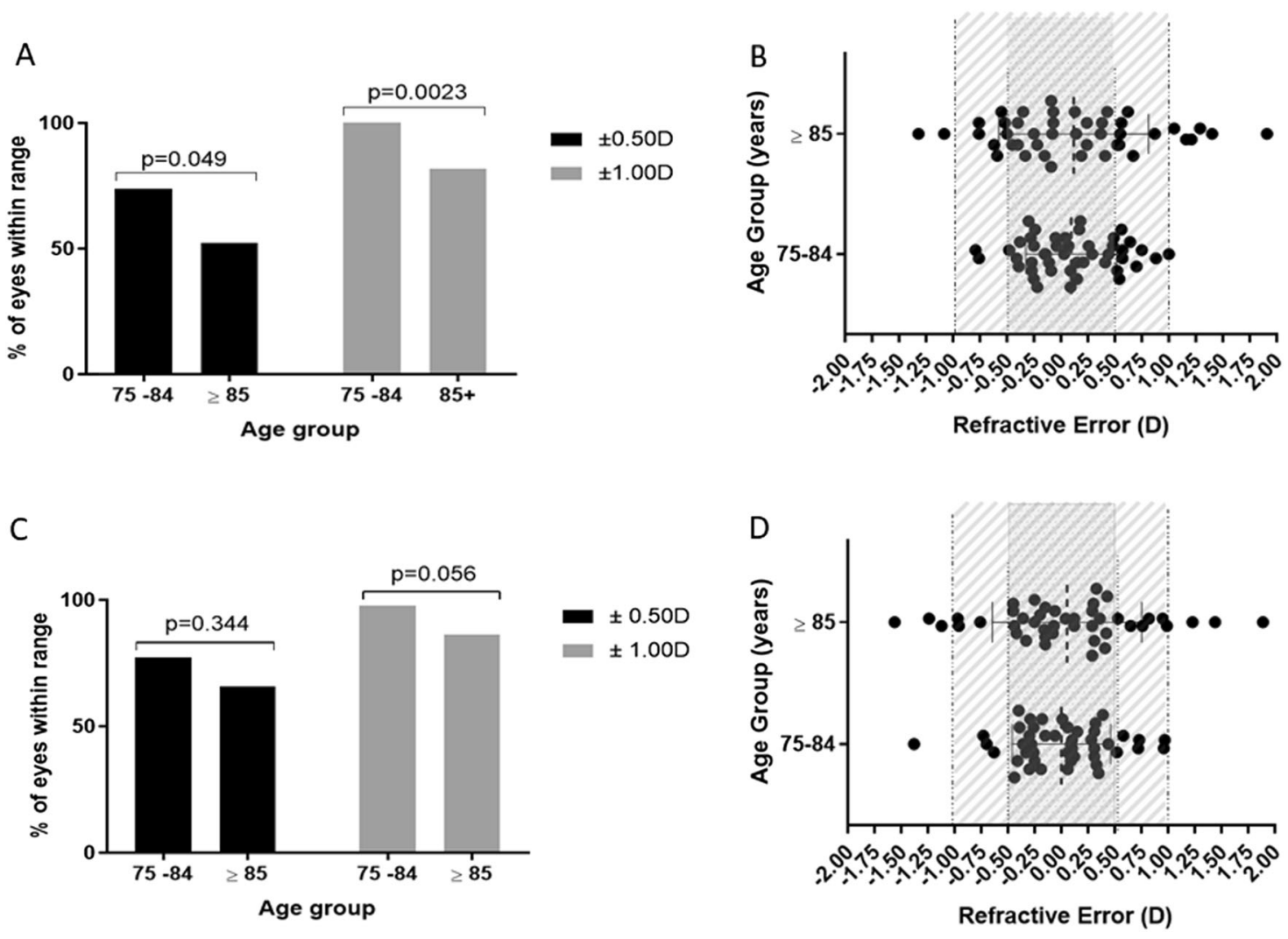

Fig. 2 Refractive outcomes within the range of $\pm 0.50 \mathrm{D}$ and $\pm 1.00 \mathrm{D}$ using the Holladay I formula and the Barrett Universal II formula among the age groups. a Percentage of eyes within a refractive outcome of $\pm 0.50 \mathrm{D}$ and $\pm 1.00 \mathrm{D}$ with the Holladay 1 formula. $\mathbf{b}$ Mean refractive error and standard deviation in both age groups with the Holladay 1 formula. Dashed lines represent the $\pm 1.00 \mathrm{D}$ border, the $\pm 0.50 \mathrm{D}$ border and the mean. Orange rectangle - area within $\pm 0.50 \mathrm{D}$.

Red striped rectangle-area within $\pm 1.00 \mathrm{D}$. c. Percentage of eyes within a refractive outcome of $\pm 0.50 \mathrm{D}$ and $\pm 1.00 \mathrm{D}$ with the Barrett Universal II formula. d Mean refractive error and standard deviation in both age groups with the Barrett Universal II formula. Dashed lines represent the $\pm 1.00 \mathrm{D}$ border, the $\pm 0.50 \mathrm{D}$ border and the mean. Orange rectangle - area within $\pm 0.50 \mathrm{D}$. Red striped rectangle — area within \pm $1.00 \mathrm{D}$.
Table 3 Multiple logistic regression analysis on $>1.00 \mathrm{D}$ absolute refractive error for Holladay I formula and the Barrett Universal II formula.

\begin{tabular}{|c|c|c|c|c|c|c|}
\hline \multirow[t]{2}{*}{ Parameter } & \multicolumn{3}{|c|}{ Holladay 1 formula } & \multicolumn{3}{|c|}{ Barrett Universal II formula } \\
\hline & $p$ value & OR & $\begin{array}{l}95 \% \text { confidence } \\
\text { interval for OR }\end{array}$ & $p$ value & OR & $\begin{array}{l}95 \% \text { confidence } \\
\text { interval for OR }\end{array}$ \\
\hline Gender & 0.22 & 0.3 & $0.04-2.06$ & 0.54 & 0.55 & $0.08-3.65$ \\
\hline Age & $0.046^{\ddagger}$ & 1.25 & $1.00-1.56$ & 0.41 & 1.08 & $0.90-1.30$ \\
\hline ACD & 0.18 & 0.16 & $0.01-2.30$ & 0.1 & 0.09 & $0.01-1.54$ \\
\hline AL & 0.83 & 0.89 & $0.30-2.61$ & 0.74 & 1.21 & $0.39-3.77$ \\
\hline Mean K & 0.93 & 0.98 & $0.54-1.75$ & 0.49 & 1.23 & $0.69-2.20$ \\
\hline
\end{tabular}

$O R$ odds ratio, $A C D$ anterior chamber depth, $A L$ axial length, mean $K$ mean keratometry

${ }^{\dagger}$ Represents $p$ value $<0.05$
Figure 2 presents the refractive outcomes within the range of $\pm 0.50 \mathrm{D}$ and $\pm 1.00 \mathrm{D}$ using the Holladay I formula and the Barrett Universal II formula. Logistic regression analysis supported the relationship between a Holladay 1 PE of $>1.00 \mathrm{D}$ and patient's age $(p=0.046)$, while sex, $\mathrm{ACD}$, mean keratometry, or $\mathrm{AL}$ were not associated (Table 3). No such relationship was found with the Barrett Universal II formula (Table 3).

\section{Discussion}

The results of this study demonstrate a higher median absolute predictive error in the very elderly in comparison to younger patients using two very popular IOL formulas, though this difference is statistically significant between the age groups only when using the Holladay 1 formula. Direct comparison between formulas in the very elderly shows 
superiority of the Barrett Universal II formula in prediction accuracy. Regression analysis supports age's contribution to a higher predictive error, and, as visible in Fig. 2, a higher standard deviation around the mean RE is present in the very elderly, with more patients outside the $\pm 0.50 \mathrm{D}$ and $\pm 1.00 \mathrm{D}$ desirable refractive goals with both formulas.

Previous studies have concluded that cataract surgery is a safe and desirable procedure at any age in the presence of visually significant cataract, with favorable visual outcomes and positive impact on quality of life and cognitive status in the very elderly $[5,8,9,11,24-26]$. As summarized in a thorough review by $\mathrm{Li}$ et al, the very elderly fare as well as younger patients across different studies, with similar complication rates when adjusting for age-related systemic and ocular comorbidities [3, 7, 8, 11]. Moreover, it may be safe to assume that patients who are undergoing cataract extraction at an older age may be in better health than average for their age as they feel well enough to choose an elective surgery. It is crucial to achieve the best possible refractive outcome while addressing this group's specific anatomical characteristics $[13,14,16,17,20]$. Prior studies do not reflect the latest techniques and technologies available at the service of cataract extraction and IOL calculations. The scarcity of such studies may stem from the relatively low number of patients undergoing cataract surgery as nonagenarians and octogenarians in the absence of visually significant ocular comorbidities.

Based on the Swedish National Cataract Register, $4 \%$ of patients undergoing cataract surgery were older than 90 years [18]. Similarly, in our study, only $6 \%$ of cataract surgeries performed at a tertiary center belonged to the very elderly age group. However, 29\% (25/86 patients undergoing phacoemulsification with implantation of SN60WF IOL) of the patients in this age group were excluded for ocular comorbidities. This makes the analysis of refractive outcome and accuracy of intraocular lens calculations formulas in this subgroup of patients challenging. Mental status and systemic comorbidities may also challenge the ability of the very elderly to cooperate with meticulous manifest refraction or fixating to achieve technically decent biometry. We therefore defined good quality of preoperative biometry and postoperative BCVA of 20/40 or better as inclusion criteria, which may have excluded even more patients but may have corrected for this possible bias. The biometry SNR comparison, showing no difference in biometry quality between groups $(p=0.28)$, reduces the chance our results are attributable to cognitive factors impacting the ability of the very elderly to hold steady fixation during biometry.

We were therefore able to summarize pre- and postoperative data of 44 eyes of 44 very elderly patients that achieved favorable visual outcomes.

Previous studies have evaluated IOL power calculation formula accuracy in the pediatric population, acknowledging anatomical differences and different biometric profiles compared to adults [27, 28]. Unique anatomical features characterize the very elderly population as well. With older age, collagen fiber structure becomes less aligned, and the scleral matrix stiffens [13, 29]. It is unclear, however, how this change translates into change of AL, if at all, or physiological/refractive consequences. In addition, ACD was shown to decrease with age [17, 20, 30]. Our study found nearly identical ACD values in the elderly $(2.89 \pm 0.36 \mathrm{~mm})$ as Hayashi et al. $(2.89 \pm 0.35 \mathrm{~mm})$, which are significantly lower than ACD in younger patients [20]. While their study also states that $\mathrm{AL}$ shortens with increasing age, no such difference was found when comparing the 70-79-year-old patients to the 80-89-year-old ones, correlating with our findings. Moreover, the angle structures change with age, with a decrease in the diameter of the SC and an increase of TM thickness [16]. Assia et al. reported a decrease in maximal zonular stretch with age and increase of zonular fragility [14]. These variations can potentially influence flexibility of the capsular bag. All aforementioned differences may impact the preoperatively estimated lens position (ELP) but are not currently taken into account when choosing the IOL formula for cataract surgeries in this age group. We are unaware of studies comparing the ELP with the actual postoperative IOL position in the very elderly. Performing the necessary ancillary exams to prove such differences in our cohort of patients was beyond the scope of our study.

A former study by Nuzzi et al. searched for risk factors that could explain deviation from emmetropia post cataract extraction. The authors concluded there is a higher RE in patients older than 73 years using the SRK II and SRK/T formulas and suggested age as a risk factor for deviation from emmetropia. Patients older than 85 years of age were excluded from analysis. Hayashi et al. [20] prospectively examined the IOL PE in younger versus older patients. In their study, four age groups (59 and younger, 60-69, 70-79, and 80-89 years of age) were compared for median absolute RE using the SRK/T formula only. The authors evaluated only eyes which were targeted for emmetropia and concluded that the mean post-operative SE was more myopic as age increased, and the mean PE was less myopic as age increased. In contrast to our results, they did not find a difference in the median absolute RE between the age groups. The authors' conclusion was, nevertheless, that age should be considered when selecting IOL power. A possible explanation for the different results is the definition of the very elderly in our study as $\geq 85$ years old, as currently accepted [1]. The formulas compared were also different, as we have compared a fourth-generation formula to the widely used Holladay 1 formula.

The Holladay 1 formula is a theoretical vergence formula. To predict the ELP, it uses two variables of 
prediction, $\mathrm{AL}$ and keratometry, and incorporates the "surgeon factor" into the formula [31]. The Holladay 1 does not take ACD into consideration, which can partially explain the differences in accuracy when used for younger versus very elderly patients. The Barrett universal II formula, on the other hand, is expected to account for differences in optical ACD, which can explain the better accuracy in this age group. The effect of ACD on the choice of intraocular lens calculation formula was previously compared between the SRK/T, Holladay 1, Hoffer $\mathrm{Q}$, and Haigis formulas in different eye lengths [32]. The Holladay 1 was inferior to the Hoffer Q formula in short eyes with an ACD shallower than $2.5 \mathrm{~mm}$, but no statistically significant difference was noted with ACD between 2.5 and $3.5 \mathrm{~mm}$. In our practice, however, as in many others across the world, the choice of formula is principally based on AL, not ACD, and the Holladay 1 and Barrett universal II serve interchangeably as formulas of choice for eyes with AL between 22-26 mm. Moreover, had the ACD been associated with RE in regression analysis, this would point to inferiority of the Holladay 1 two-variable formula in eyes with shorter ACD. However, the significant association of age alone to refractive outcome by logistic regression, and the similar Barrett trend toward poorer refractive outcomes in the older age group, suggest patient's age as an important variable.

This study has a few limitations. To comply with the recommendations of Hoffer et al. for studies of IOL calculation formulas accuracy and to avoid errors of optimization, only one IOL model was hereby analyzed [21, 22]. While the 1-piece SN60WF is one of the most commonly used IOLs in use in the US, our results may not be extrapolatable to other IOL types. Additionally, given the scarcity of patients who met our inclusion criteria, surgeries by multiple surgeons were included in the analysis. Lastly, only the Barrett Universal II and the Holladay 1 formulas were compared based on their accuracies and popularity of use, especially in eyes $22-26 \mathrm{~mm}$ long [33]. The above, however, may very well represent real-life experiences of practices worldwide.

As future studies search for rationale for the differences in refractive outcomes between age groups, it would be interesting to compare lens thickness and grading of cataract in both groups prior to surgery, as well as the IOL stable postoperative anatomical location using anterior segment optical coherence tomography. It would also be of interest to investigate correlation of ACD and lens thickness with the prediction of refractive outcome in a larger series of patients. In our study, we wanted to compare the very elderly group to another age group, and chose the one of 75-84, assuming a manifest difference between two consecutive age groups may signify an even more noticeable difference with younger patients as well. Fortunately, we found a statistically significant difference with this comparison, however, comparing the refractive outcomes of the very elderly to younger age groups, specifically patients aged $65-75$ years, would be of value in the future as well.

In conclusion, this preliminary study points at a more predictable refractive outcome in younger versus very elderly patients undergoing cataract extraction, with more accurate refractive results achieved and a lower median absolute RE achieved using the Barrett Universal II formula in this age group. This should be taken into consideration when an IOL calculation formula is chosen in the very elderly and during preoperative patient consult, if the Holladay 1 formula is to be used. In a following larger cohort study, a best-fit regression equation will be obtained to improve the predictability of the Holladay 1 formula for this age group.

\section{Summary}

\section{What was known before}

- The number of cataract surgeries performed in the very elderly (over the age of 85) is on the rise.

- Refractive outcomes are not frequently analysed in this subgroup of elderly patients and none have investigated the use of modern intraocular lens calculation formulas in this age group.

\section{What this study adds}

- This study evaluates the intraocular lens calculations accuracy in this age group.

- Very elderly patients undergoing cataract surgery may be prone to reduced refractive precision.

- Predicting refractive outcomes in the very elderly may improve with the Barrett Universal II formula.

Acknowledgements All authors contributed to the study conception and design. Material preparation, data collection, and analysis were performed by RS, LC, AKS, and HEG. The first draft of the paper was written by RS and all authors commented on previous versions of the paper. All authors read and approved the final paper. The authors would like to acknowledge Saurabh Sawney and Ashima Aggrawal for the complimentary use of the online lens formula performance audit calculator, available online at http://saurabhsawhney.wix.com/calculators.

Funding This project was partially supported by the National Institutes of Health, Grant T35 AG026757/AG/NIA, an unrestricted grant from Research to Prevent Blindness (New York, NY) and the University of California San Diego, Stein Institute for Research on Aging. 


\section{Compliance with ethical standards}

Conflict of interest Financial disclosure(s): RS, LC, AKS, HEG, NAA -no relevant financial disclosures. RNW: Consultant: Aerie Pharmaceuticals, Alcon, Allergan, Bausch \& Lomb, Eyenovia, Novartis, Sensimed, Unity, Valeant; Financial support: Heidelberg Engineering, Carl Zeiss Meditec, Genentech, Konan, Optovue, Topcon, Optos, Centervue. AKS: non-financial support: Heidelberg Engineering, grants: Bayer Vital, Novartis.

Publisher's note Springer Nature remains neutral with regard to jurisdictional claims in published maps and institutional affiliations.

\section{References}

1. Suzman R, Beard J. Global health and aging presented by the National Institute on Aging, National Institutes of Health, U.S. Department of Health and Human Services. 2011. http://www. who.int/ageing/publications/global_health.pdf.

2. Foreman KJ, Marquez N, Dolgert A, Fukutaki K, Fullman N, McGaughey M, et al. Forecasting life expectancy, years of life lost, and all-cause and cause-specific mortality for 250 causes of death: reference and alternative scenarios for 2016-40 for 195 countries and territories. Lancet. 2018;392:2052-90.

3. Mutoh T, Isome S, Matsumoto Y, Chikuda M. Cataract surgery in patients older than 90 years of age. Can J Ophthalmol. 2012;47:140-4.

4. Lundström M, Stenevi U, Thorburn W. Cataract surgery in the very elderly. J Cataract Refract Surg. 2000;26:408-14.

5. Tseng VL, Greenberg PB, Wu W-C, Jiang L, Li E, Kang JM, et al. Cataract surgery complications in nonagenarians. Ophthalmology. 2011;118:1229-35.

6. Toyama T, Ueta T, Yoshitani M, Sakata R, Numaga J. Visual acuity improvement after phacoemulsification cataract surgery in patients aged $\geq 90$ years. BMC Ophthalmol. 2018;18:280.

7. Rosen E, Rubowitz A, Assia EI. Visual outcome following cataract extraction in patients aged 90 years and older. Eye. 2009;23:1120-4.

8. Mönestam E, Wachmeister L. Impact of cataract surgery on the visual ability of the very old. Am J Ophthalmol. 2004;137: 145-55.

9. Song E, Sun H, Xu Y, Ma Y, Zhu H, Pan C-W. Age-related cataract, cataract surgery and subsequent mortality: a systematic review and meta-analysis. PLoS One. 2014;9:e112054.

10. Robbie SJ, Muhtaseb M, Qureshi K, Bunce C, Xing W, Ionides A. Intraoperative complications of cataract surgery in the very old. $\mathrm{Br}$ J Ophthalmol. 2006;90:1516-8.

11. Li E, Margo CE, Greenberg PB. Cataract surgery outcomes in the very elderly. J Cataract Refract Surg. 2018;44:1144-9.

12. Lai FHP, Lok JYC, Chow PPC, Young AL. Clinical outcomes of cataract surgery in very elderly adults. J Am Geriatr Soc. 2014;62:165-70

13. Coudrillier B, Pijanka J, Jefferys J, Sorensen T, Quigley HA, Boote $\mathrm{C}$, et al. Effects of age and diabetes on scleral stiffness. J Biomech Eng. 2015;137:071007.

14. Assia EI, Apple DJ, Morgan RC, Legler UF, Brown SJ. The relationship between the stretching capability of the anterior capsule and zonules. Investig Ophthalmol Vis Sci. 1991; $32: 2835-9$.
15. Michael R, Mikielewicz M, Gordillo C, Montenegro GA, Pinilla Cortés L, Barraquer RI. Elastic properties of human lens zonules as a function of age in presbyopes. Investig Ophthalmol Vis Sci. 2012;53:6109-14.

16. Chen Z, Sun J, Li M, Liu S, Chen L, Jing S, et al. Effect of age on the morphologies of the human Schlemm's canal and trabecular meshwork measured with swept-source optical coherence tomography. Eye. 2018;32:1621-8.

17. Hashemi H, Yekta A, Khodamoradi F, Aghamirsalim M, Asharlous A, Assadpour M, et al. Anterior chamber indices in a population-based study using the Pentacam. Int Ophthalmol. 2019;39:2033-40.

18. Behndig A, Montan P, Stenevi U, Kugelberg M, Lundström M. One million cataract surgeries: Swedish National Cataract Register 1992-2009. J Cataract Refract Surg. 2011;37:1539-45.

19. Nuzzi G, Cantù C, De Giovanni MA. Older age as risk factor for deviation from emmetropia in pseudophakia. Eur J Ophthalmol. 2001;11:133-8.

20. Hayashi K, Ogawa S, Yoshida M, Yoshimura K. Influence of patient age on intraocular lens power prediction error. Am J Ophthalmol. 2016;170:232-7.

21. Hoffer KJ, Aramberri J, Haigis W, Olsen T, Savini G, Shammas $\mathrm{HJ}$, et al. Protocols for studies of intraocular lens formula accuracy. Am J Ophthalmol. 2015;160:403-.e1.

22. Hoffer KJ. Accuracy of ultrasound intraocular lens calculation. Arch Ophthalmol. 1981;99:1819-23.

23. Wang L, Tang M, Huang D, Weikert MP, Koch DD. Comparison of newer intraocular lens power calculation methods for eyes after corneal refractive surgery. Ophthalmology. 2015;122 (Dec):2443-9.

24. Fraser M, Meuleners L, Bulsara M, Van DuongD, Van DoD, Huynh V-AN, et al. A longitudinal cohort study of the impact of first- and both-eye cataract surgery on falls and other injuries in Vietnam. Clin Inter Aging. 2014;9:743.

25. Yu W-K, Chen Y-T, Wang S-J, Kuo S-C, Shia B-C, Liu CJ-L. Cataract surgery is associated with a reduced risk of dementia: a nationwide population-based cohort study. Eur J Neurol. 2015;22:1370-7. e79-80

26. Jefferis JM, Clarke MP, Taylor J-P. Effect of cataract surgery on cognition, mood, and visual hallucinations in older adults. $\mathrm{J}$ Cataract Refract Surg. 2015;41:1241-7.

27. Lee BJ, Lee S-M, Kim JH, Yu YS. Predictability of formulae for intraocular lens power calculation according to the age of implantation in paediatric cataract. Br J Ophthalmol. 2018. https:// doi.org/10.1136/bjophthalmol-2017-311706.

28. Neely DE, Plager DA, Borger SM, Golub RL. Accuracy of intraocular lens calculations in infants and children undergoing cataract surgery. J AAPOS. 2005;9:160-5.

29. Fazio MA, Grytz R, Morris JS, Bruno L, Gardiner SK, Girkin CA, et al. Age-related changes in human peripapillary scleral strain. Biomech Model Mechanobiol. 2014;13:551-63.

30. Hoffer KJ. Biometry of 7,500 cataractous eyes. Am J Ophthalmol. 1980;90:360-8.

31. Holladay JT, Prager TC, Chandler TY, Musgrove KH, Lewis JW, Ruiz RS. A three-part system for refining intraocular lens power calculations. J Cataract Refract Surg. 1988;14:17-24.

32. Yang S, Whang W-J, Joo C-K. Effect of anterior chamber depth on the choice of intraocular lens calculation formula. PLoS ONE. 2017;12:e189868.

33. Melles RB, Holladay JT, Chang WJ. Accuracy of intraocular lens calculation formulas. Ophthalmology. 2018;125:169-78. 